\title{
INFLUENCIA DEL GENOTIPO Y FECHA DE SIEMBRA EN LA CALIDAD DE SEMILLA DE SORGO INFECTADA CON ERGOT
}

\section{GENOTYPE AND PLANTING DATE EFFECTS ON THE QUALITY OF SORGHUM SEEDS INFECTED WITH ERGOT}

\author{
Ma. Eugenia Cisneros-López ${ }^{1}$ y Leopoldo E. Mendoza-Onofre ${ }^{2}$
}

\begin{abstract}
${ }^{1}$ Campo Experimental Río Bravo, Instituto Nacional de Investigaciones Forestales, Agrícolas y Pecuarias. Km. 61 Carretera Matamoros-Reynosa. 88900, Río Bravo, Tamaulipas. ${ }^{2}$ Postgrado en Recursos Genéticos y Productividad-Producción de Semillas, Colegio de Postgraduados, Campus Montecillo. 56230, Montecillo, Texcoco, Estado de México.
\end{abstract}

* Autor para correspondencia (maya1163@prodigy.net.mx)

\section{RESUMEN}

El "ergot" o cornezuelo es una enfermedad causada por el hongo Claviceps africana Frederikson, Mantle \& de Milliano que origina pérdidas en el rendimiento de semilla de sorgo (Sorghum bicolor $\mathbf{L}$. Moench). Además, la semilla producida por las flores adyacentes a las infectadas se contamina con el exudado del patógeno y se coloniza por hongos saprofitos, que disminuyen la calidad. En este estudio se evaluó la germinación, viabilidad, vigor, conductividad eléctrica, peso y calidad sanitaria de semilla de dos líneas androestériles sembradas en mayo y junio de 2006 en Montecillo, Edo. de México (2200 msnm), con y sin fungicida (Thiram (8), combinados con tres grados de severidad de infección a la panoja $(0,10$ y $50 \%)$ causada por el patógeno, en condiciones de infección natural en campo. A los $8 \mathrm{~d}$ después de la cosecha, el genotipo y la fecha de siembra tuvieron mayor efecto $(P \leq 0.05)$ que el fungicida $y$ la severidad de $C$. africana en la calidad física y fisiológica de la semilla. La semilla de la línea androestéril A1 tuvo mejor calidad, germinación y vigor que la línea A9. La semilla del lote sembrado el 5 de mayo fue mejor que la del 10 de junio. En promedio, hubo $31 \%$ menos colonias fungosas en la semilla tratada con fungicida que sin éste y las semillas de panojas sanas tuvieron la mitad de colonias que las que presentaron $50 \%$ de severidad. En general, la calidad sanitaria de la semilla fue afectada por el grado de daño a la panoja causada por el ergot en campo, pero no por la aplicación de fungicida a la semilla.

Palabras clave: Claviceps africana, Sorghum bicolor, conductividad, germinación, vigor de semilla.

\section{SUMMARY}

Ergot (Claviceps africana Frederickson, Mantle \& de Milliano) is a disease that decreases sorghum (Sorghum bicolor L. Moench) seed yield. Moreover, seeds near infected flowers become contaminated with honeydew and exudates thus enhancing saprophytic fungus colonization and decreasing seed quality. Germination, viability, vigor, electrical conductivity, weight and sanitary quality of seed from two male-sterile sorghum lines were evaluated. Lines were sown in two planting dates (May and June 2006,) in Montecillo, State of México $(2200 \mathrm{~m}$ altitude). Treated and non-treated seeds with fungicide (Thiram $\left.{ }^{\circledR}\right)$, in combination with three levels of $C$. africana severity (0, 10 y $50 \%)$ on panicles naturally infected under

Recibido: 15 de Diciembre del 2008.

Aceptado: 18 de Enero del 2010. field conditions, were also compared. Eight days after harvest, genotype and sowing date showed higher effects $(P \leq 0.05)$ than fungicide application and $C$. africana severity, on the physical and physiological seed quality traits. Seed quality, germination and vigor of the line A1 were superior to the corresponding values of line A9. Seed from plots sown in May 5th had higher quality than those sown in June 10th. On the average, fungicide treated seed had $31 \%$ less fungus colonies than non-treated seed, while seeds from healthy panicles had half of the colonies than those showing $50 \%$ of ergot severity. In general, sanitary quality was affected by the ergot damage in the field but not by the fungicide treatment.

Index words: Claviceps africana, Sorghum bicolor, conductivity, germination, seed vigor.

\section{INTRODUCCIÓN}

En México, el sorgo granífero (Sorghum bicolor L. Moench) ocupa el segundo lugar en importancia agrícola por la magnitud de su producción (3.3 millones de toneladas) y el tercer sitio en superficie sembrada (un millón de hectáreas), con un rendimiento promedio nacional de $3.8 \mathrm{t} \mathrm{ha}^{-1}$ (SIAP, 2008). Los problemas sanitarios del sorgo son la segunda causa de pérdidas en el rendimiento, después de la sequía. El "ergot", "enfermedad azucarada" o "cornezuelo" causado por Claviceps africana (Frederickson, Mantle \& de Milliano), ataca a todos híbridos comerciales de sorgo, con incidencias hasta de $100 \%$, y con grados de severidad entre 1 y $10 \%$ (Montes-Belmont et al., 2002). El patógeno es particularmente perjudicial en lotes de producción de semilla híbrida pues las líneas androestériles son más susceptibles que las androfértiles (Meinke y Ryley, 1997); i.e., en el Bajío se han registrado mermas de 45 a $56 \%$ en rendimiento, con incidencias de 42 a $66 \%$ y severidades de 19 a $22 \%$ (Hernández-Martínez et al., 2006); en el Norte de Tamaulipas las reducciones del rendimiento han variado de 30 a $100 \%$ (Montes-García et 
al., 2009). La magnitud del daño originó que se estableciera una Norma Oficial Mexicana (NOM) para la producción e importación de semilla (Odvody et al., 2002).

El hongo afecta pistilos no polinizados; sus hifas colonizan el ovario y sustituyen al grano por una masa compacta y endurecida (esclerocio) (Frederiksen y Odvody, 2000). En forma indirecta, el hongo disminuye la calidad física, fisiológica y sanitaria de la semilla (Hernández-Martínez et al., 2006), pues ésta se contamina con exudados del patógeno lo que favorece la colonización por hongos saprófitos (Bandyopadhyay et al., 1998), inhibe la germinación de la semilla y el crecimiento de plántulas, y aumenta la predisposición a enfermedades como la pudrición de plántulas ("damping off") (McLaren, 1993); la presencia de exudados también dificulta la cosecha (Bandyopadhyay et al., 1990).

El vigor de la semilla depende de la constitución genética de la planta madre y del ambiente biótico y abiótico que la rodea, desde su germinación y posterior crecimiento de la plántula, hasta la cosecha. Las condiciones ambientales imperantes durante la etapa postcosecha y en el almacenamiento también influyen en el vigor de la semilla (Marsans, 1987; Shekaramúrthy et al., 1994). El disulfuro de tetrametiltiuram (Thiram $\left.{ }^{\circledR}\right)$ y el (3a, 4, 7, 7 a-tetrahidro-2-[(triclorometil) tioéter]-1Hisoindol-1, $3(2 \mathrm{H})$-di) (Captán 400®) son fungicidas de contacto que se recomiendan aplicar durante el acondicionamiento de la semilla de sorgo (Vidhyasekaran, 1983), para prevenir la esporulación de conidios en el exudado de $C$. africana adherido a la semilla (Dahlberg et al., 1999). Además, Shekaramúrthy et al. (1994) indican que Thiram ${ }^{\circledR}$ fue más efectivo para controlar hongos de campo (como Alternaria y Phoma) que hongos de almacén (Aspergillus). En México no se dispone de información acerca del efecto del tratamiento químico en la semilla de sorgo infectada con C. africana.

El objetivo de este estudio fue evaluar el efecto de dos fechas de siembra, tres grados de severidad a la panoja causado por la incidencia natural de $C$. africana en campo y el tratamiento químico a la semilla, en la germinación, viabilidad, vigor, peso, conductividad eléctrica y sanidad de la semilla de dos líneas androestériles de sorgo.

\section{MATERIALES Y MÉTODOS}

En Montecillo, Edo. de México ( $18^{\circ} 56^{\prime} 4^{\prime \prime} \mathrm{LN}, 97^{\circ}$ 49' 54' 'LO, $2200 \mathrm{~m}$ de altitud) se establecieron dos lotes (Lote 1, 5 de mayo; Lote 2, 10 de junio de 2006), en condiciones de riego. Cada lote se dividió en dos secciones; en una se incrementó semilla de la línea A1 mediante cruzamiento con su línea isogénica B1 (o sea, A1xB1); en la otra sección se ubicó a la línea A9 (A9xB9). En ambos casos la polinización fue libre. Para evitar contaminación de polen ajeno a la cruza de interés, cada sección se aisló con al menos 10 surcos de maíz (Zea mays L.). En ambos lotes la incidencia natural de $C$. africana fue semejante. Quince días después de la floración, las panojas de las líneas androestériles se cubrieron con bolsas de papel para polinizar con objeto de evitar daño por pájaros. Las panojas se mantuvieron en esta condición por $40 \mathrm{~d}$ en el Lote $1 \mathrm{y}$ por $48 \mathrm{~d}$ en el Lote 2 , hasta la cosecha manual el 5 de octubre y 8 de noviembre, respectivamente, cuando el grano tenía $13 \%$ de humedad. A la cosecha, se evaluó en forma visual la severidad del daño causado por $C$. africana a la panoja (glumas con exudado, o presencia de micelio, esclerocios o ambos) (Hernández-Martínez et al., 2006). Las panojas con grados de severidad de 0,10 y $50 \%$ (máximo valor observado) se agruparon, se desgranaron individualmente y la semilla se cribó (criba oblonga de 6/64", $2.3 \mathrm{~mm}$ ). La temperatura ambiental y humedad relativa se registraron durante el ciclo del cultivo en una estación meteorológica digital (ADCON® ${ }^{\circledR}$ Mod. 840), localizada a $150 \mathrm{~m}$ de cada lote.

Ocho días después de la cosecha se formó una muestra compuesta por genotipo, fecha de siembra y grado de severidad $(\mathrm{S} 0=0 \%, \mathrm{~S} 10=10 \%$ y S50 $=50 \%)$. De inmediato, se aplicó Thiram ${ }^{\circledR}$ (dosis comercial equivalente a $0.1 \mathrm{~g}$ de i. a. $/ \mathrm{kg}$ de semilla), a la mitad de la muestra. El testigo fue la semilla sin infección del patógeno y sin tratamiento químico. Una semana después de que se aplicó el fungicida se midieron las siguientes variables:

Germinación y vigor. Se aplicó la metodología aprobada por ISTA (1999) que consiste en sembrar tres repeticiones de 100 semillas cada una, en toallas de papel. Al cuarto día (técnica al primer conteo) se evaluó el vigor $(\mathrm{V}=$ semillas que germinan con estructuras completas y sanas: radícula, mesocótilo alargado y coleóptilo). Siete días después de la siembra se contó el número de plántulas normales $(\mathrm{PN}=$ con sistema radicular bien desarrollado, mesocótilo alargado y hojas primarias verdes, intactas, expandidas y sanas), plántulas anormales $(\mathrm{PA}=$ con ausencia, deformación o enfermedad en alguna de esas estructuras) y semillas muertas (PSM = sin estructura alguna) (Bekendam y Grob, 1980; ISTA, 1999). La germinación se basó solamente en plántulas normales. Estas variables se expresaron en porcentaje.

Viabilidad (VIA). Tres repeticiones de 100 semillas cada una se sumergieron en agua por $12 \mathrm{~h}$; después las semillas se cortaron longitudinalmente y el embrión se expuso a una solución de cloruro de tetrazolio. La mitad 
de esta semilla se usó para la prueba y la otra mitad se desechó (ISTA, 1999). Los resultados se expresaron en porcentaje de semillas viables.

Peso de 100 semillas (P100S). Se tomaron al azar tres repeticiones de 100 semillas y se pesaron en una balanza Ohaus Mod. 5000®. Los resultados se expresaron en gramos $(\mathrm{g})$.

Conductividad eléctrica (C). Se pesaron y colocaron 50 semillas en un vaso de cristal de $250 \mathrm{~mL}$ con tres repeticiones por tratamiento. Posteriormente se incubaron por $24 \mathrm{~h}$ a $25{ }^{\circ} \mathrm{C}$ en $50 \mathrm{~mL}$ de agua deionizada y destilada. Se retiró la semilla y se determinó la conductividad del agua con un conductímetro (Oakton ${ }^{\circledR}$, Mod WD-35607-00). Los resultados se expresaron en microsiemens por gramo de semilla $\left(\mu \mathrm{s} \mathrm{g}^{-1}\right)$.

Sanidad. La semilla de cada tratamiento se desinfestó con una solución comercial de hipoclorito de sodio a $6 \%$. Posteriormente, se sembraron 10 semillas en una caja Petri que contenía un medio sólido de PDA (papa, dextrosa, agar) a $50 \%$ (tres repeticiones por tratamiento, una caja por repetición). Las cajas se mantuvieron en incubación con luz continua durante $8 \mathrm{~d}$. Al quinto día se tomaron muestras del micelio para la identificación de especies como proponen Wen-Shi y Kuang-Che (1990) y Warham et al. (1994). La calidad sanitaria se cuantificó por el número promedio de colonias fungosas por tratamiento porque el ergot, como patógeno primario, favorece la presencia de micoflora secundaria que se presenta durante la etapa de postfloración y postcosecha.

\section{Análisis estadísticos}

A cada variable cuantitativa se le aplicó un análisis de varianza (SAS Institute, 2002) con el diseño completamente al azar balanceado, en arreglo factorial $2 \times 2 \times 2 \times$ 3 para medir el efecto de dos fechas de siembra (F1: mayo y F2: junio de 2006), dos genotipos (G: A1 y A9), la aplicación de fungicida a la semilla $(\mathrm{Q}$ : con y $\sin ) \mathrm{y}$ tres grados de severidad de C. africana (S: 0,10 y 50 $\%)$ así como sus respectivas interacciones. La comparación de medias fue mediante la prueba de Tukey $(P=0.05)$. Además, se hicieron correlaciones simples de Pearson entre porcentaje de plántulas normales, anormales, muertas, viabilidad, vigor, P100S, conductividad eléctrica y número de colonias fungosas. Se aplicó la prueba de $\mathrm{t}$ de Student para comparar los contrastes entre: línea A1 vs. A9; F1 vs. F2; con vs. sin fungicida, y entre grados de severidad: $\mathrm{S} 0 v s$. S10, S0 vs. S50 y S10 vs. S50 para la variable número de colonias fungosas.

\section{RESULTADOS Y DISCUSIÓN Calidad fisiológica y física de la semilla}

Genotipo y fecha de siembra tuvieron efectos significativos $(\mathrm{P} \leq 0.05)$ en todas las variables con excepción del genotipo en el peso de 100 semillas (tamaño de la semilla) y de la fecha de siembra en el porcentaje de plántulas anormales (Cuadro 1). En contraste, la aplicación del fungicida no influyó en estas variables, a diferencia de la severidad del daño de $C$. africana que las afectó a todas. Con base en la magnitud de los cuadrados medios de cada factor individual, el genotipo y la fecha de siembra tuvieron mayor efecto (en promedio explicaron entre 14 y $32 \%$ de la varianza total) que el fungicida y la severidad (los cuales oscilaron entre $<0.40$ y $12 \%$ ); incluso el efecto individual de al menos uno de ellos superó al de cualquiera de las interacciones en las siete variables. Estos resultados confirman que los factores ambientales (inherentes a la fecha de siembra) y genéticos determinan la condición fisiológica de la semilla porque influyen desde la formación hasta la madurez de la misma.

La calidad de la semilla de la línea A1 fue mejor que la de la línea A9 pues la primera tuvo mayor germinación, viabilidad y vigor, y menor conductividad que la segunda (Cuadro 2). La conductividad eléctrica de la semilla es una forma indirecta de medir el vigor de la semilla (ISTA, 1999): a mayor conductividad menor vigor. La línea A1 también tuvo menor cantidad de semillas muertas, aunque la diferencia con A9 fue de sólo dos puntos porcentuales. Ambas líneas cumplieron con la germinación $(80 \%)$ requerida por la NOM para semilla de sorgo (SNICS, 1987) de alto registro (básica y registrada). No se dispone de información bibliográfica acerca de las diferencias de textura del endospermo y demás características físicas o fisiológicas que pudieran explicar las diferencias entre las semillas de ambas líneas androestériles; sin embargo, en las líneas mantenedoras B1 y B9 el peso del endospermo, del embrión y de la proporción endospermo/embrión fue similar (ValadezGutiérrez et al., 2006). En maíz no se observó efecto significativo del tipo de endospermo (harinoso o cristalino) en la germinación y vigor de semilla recién cosechada (Woltz et al., 2006); además, en maíz los cambios de germinación y vigor entre una línea tolerante y otra susceptible al almacenamiento se han atribuido a las diferencias fisiológicas en el endospermo y eje embrionario (Cruz-Pérez et al., 2002). 

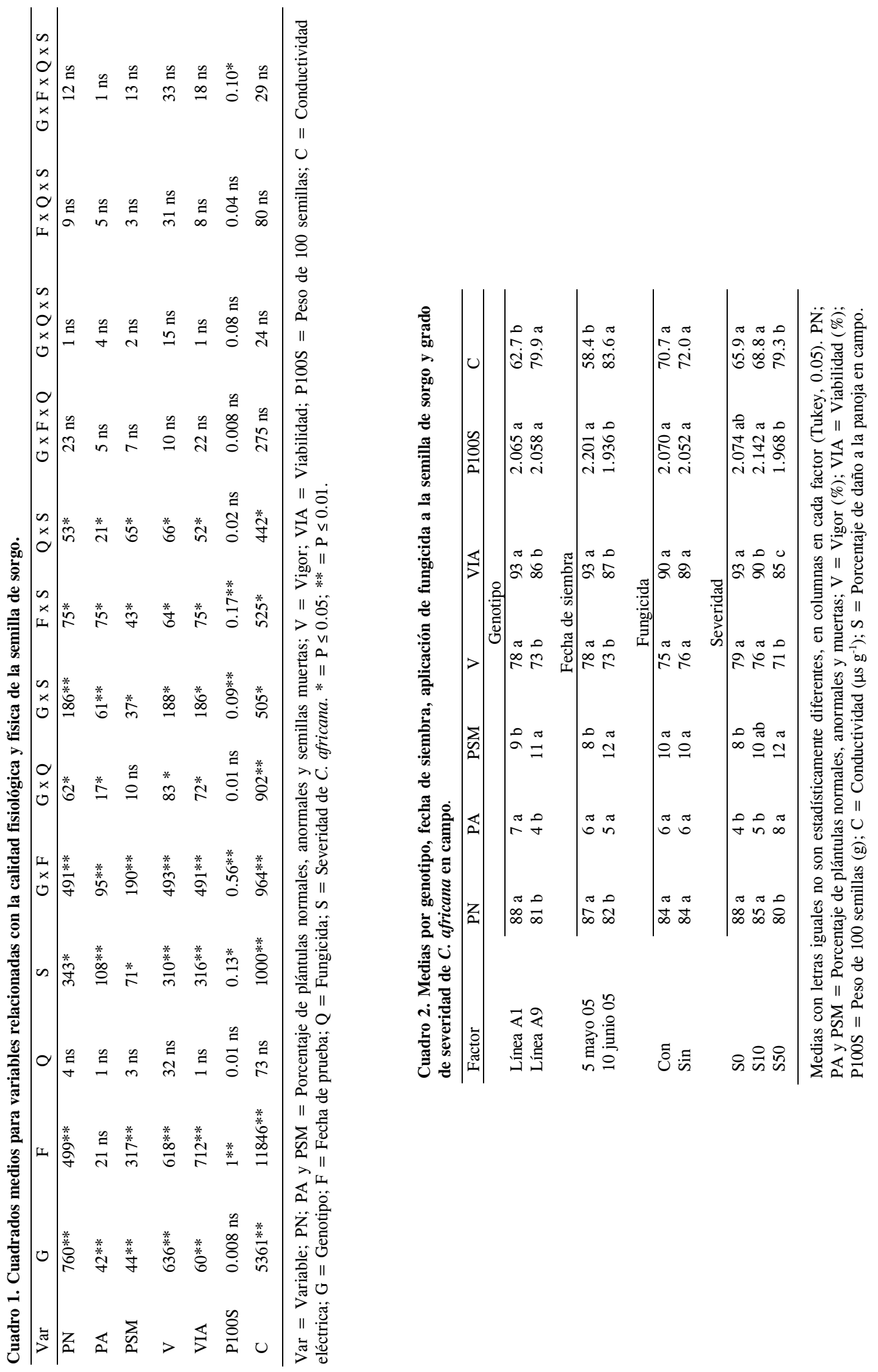
El porcentaje de semillas muertas varió de 8 a 12 , mientras que el de plántulas anormales fluctúo de 4 a $8 \%$ (Cuadro 2). Las semillas muertas permiten la salida de iones del interior de la célula, ya que las membranas pierden su integridad (Veselova y Veselovsky, 2003) lo que origina un aumento en la conductividad eléctrica (Powell, 1988).

Entre fechas de siembra, la semilla de mejor calidad provino del lote sembrado el 5 de mayo porque presentó mayores valores de germinación (87 \%), vigor (78 \%), viabilidad (93 \%), y tamaño de semilla $(2.20 \mathrm{~g}$ de 100 semillas), así como menor conductividad eléctrica (58.4 $\mu \mathrm{s}^{-1}$ ) que la que se produjo en la siembra del 10 de junio (82, 73 y $87 \%$; 1.93 g y $83.6 \mu \mathrm{s} \mathrm{g}^{-1}$ respectivamente). Se han reportado diferencias entre fechas de siembra en la germinación estándar (plántulas normales), velocidad de germinación (primer conteo) y conductividad eléctrica de semillas del pasto Sudán [(Sorghum bicolor (L.) Moench subsp. drummondii] (Wang et al., 2004).

En el presente experimento no hubo correlación significativa $(r=0.18$ a 0.68$)$ entre el peso de 100 semillas y porcentaje de plántulas normales y anormales, vigor, viabilidad y conductividad eléctrica, pero sí con el porcentaje de semillas muertas $\left(\mathrm{r}=-0.78^{*}\right)$ (Cuadro 4). Cisneros-López et al. (2007) tampoco encontraron correlación entre el peso de 100 semillas y el porcentaje de plántulas normales, anormales, semillas muertas y velocidad de germinación de semillas de sorgo almacenadas durante ocho años. Sin embargo, en semilla de maíz forrajero se encontró correlación entre el tamaño de la semilla con su calidad fisiológica (Pérez et al., 2006). El peso de la semilla es importante porque se define durante el periodo de llenado de grano, aunque el número potencial de semillas se genera durante el desarrollo floral; es decir, esta variable refleja las condiciones de crecimiento del cultivo (Peña et al., 2001) y constituye la reserva potencial de la semilla durante la germinación. En el presente experimento no hubo diferencias entre genotipos en peso de 100 semillas (Cuadro 2).

La aplicación del fungicida no modificó $(\mathrm{P} \leq 0.05)$ la calidad fisiológica ni física de la semilla recién cosechada (Cuadro 2). En cacahuate (Arachis hipogaea L.) la aplicación del fungicida Captán ${ }^{\circledR}$ mejoró la germinación pero no el vigor de la semilla, en comparación con semilla sin tratar (Narasimhulu y Rao, 1989); similar respuesta se observó con aplicación de Thiram ${ }^{\circledR}$ en semilla de sorgo sometida a envejecimiento acelerado (Shekaramúrthy et al., 1994). En semilla de frijol (Phaseolus vulgaris L.) tratada con Captán ${ }^{\circledR}$ y Benomyl ${ }^{\circledR}$ para prevenir la infección natural de Fusarium spp., se han reportado diferencias entre genotipos (NavarreteMaya y Acosta-Gallegos, 1999). En semilla de sorgo infestada por C. africana, Dahlberg et al. (1999) no encontraron diferencias significativas en la germinación de la semilla cuando aplicaron Thiram ${ }^{\circledR}$ o Captán ${ }^{\circledR}$, respecto al testigo sin tratamiento; a pesar de eso, ambos productos se recomiendan porque pueden inhibir la esporulación de conidios inmersos en el exudado fungoso. Este mismo efecto tiene el Captán ${ }^{\circledR}$ cuando se aplica a los esfacelios y esclerocios que mezclados con la semilla (Frederikson y Odvody, 2003). El propósito del fungicida aplicado a la semilla después de la cosecha, es controlar el complejo de patógenos que impiden a la semilla emerger y a la plántula establecerse en campo, y no necesariamente controlar solamente a un patógeno, (i.e., ergot (Dabkeviĉius y Mikaliūnaitê, 2006). Ante estas diferencias, es evidente que se requieren más estudios acerca del efecto de la aplicación de fungicidas a la semilla, así como de sus posibles interacciones con el genotipo, patógeno, y con los tiempos de almacenamiento. En las variables relacionadas con la calidad fisiológica de la semilla, el fungicida presentó interacciones de distinto orden y magnitud con los factores: genotipo, fecha de siembra y severidad del ergot, aunque el peso estadístico del fungicida per se fue menor a $1 \%$ de la varianza total (Cuadros 1 y 3 ).

La severidad del daño que $C$. africana causa a la panoja del sorgo tuvo efecto significativo en todas las variables (Cuadro 1), lo cual refleja la importancia del deterioro que el hongo causa en la calidad postcosecha de la semilla. En las panojas en las que la severidad fue mayor (50 \%) hubo mayor deterioro de la semilla; i.e., al considerar al testigo (sin daño) como referente, la germinación, viabilidad y vigor se redujeron en ocho puntos porcentuales y el peso de 100 semillas en $5 \%$; la salida de solutos por incremento de la conductividad eléctrica aumentó en $20 \%$ y las semillas muertas se incrementó en $4 \%$ (Cuadros 2 y 3). En la línea de sorgo 'E15A' con severidad por ergot de $20 \%$, Hernández-Martínez et al. (2006) reportaron que en las panojas contaminadas disminuyó la proporción de semilla de tamaño grande (cribas de $3.6 \mathrm{~mm}$ ), la viabilidad y la germinación de la semilla; aumentó la proporción de semilla de tamaño menor (cribas de $2.4 \mathrm{~mm}$ ). Además, las infecciones tempranas en el grano de sorgo causadas por hongos patogénicos generalmente acortan el periodo de llenado de grano (Castor y Fredericksen, 1981) con la consecuente pérdida de peso (Singh y Makne, 1985). La reducción en la germinación y el aumento de plántulas anormales y semillas muertas (Cuadros 2 y 3 ) se puede atribuir al efecto tóxico de los exudados del patógeno que inhiben la germinación y el vigor (McLaren, 1993), y a la presencia 
de patógenos secundarios (como Alternaria spp.) que colonizan el embrión (McLaren et al., 2002).

El efecto en la calidad de la semilla de la fecha de siembra y de sus interacciones de primer grado con el genotipo $(\mathrm{G} \times \mathrm{F})$ y con el grado de daño a la panoja ( $\mathrm{F} x$ S) (Cuadros 1, 2 y 3) tienen importancia biológica y fitopatológica, ya que la incidencia y severidad de $C$. africana están correlacionadas con la temperatura y humedad relativa (Bandyopadhyay et al., 1998), y varían con la fecha de siembra (Montes-García et al., 2009). En inoculación artificial, las condiciones óptimas para lograr la infección son humedades relativas cercanas a $100 \%$ y temperatura de $19.5^{\circ} \mathrm{C}$ en un rango de 14 a $28{ }^{\circ} \mathrm{C}$, con un límite máximo de $30{ }^{\circ} \mathrm{C}$ (Futrell y Webster, 1966; McLaren y Wehner, 1990). En el norte de Tamaulipas, México, en condiciones naturales de infección las temperaturas favorables variaron de 13.3 a $26.7^{\circ} \mathrm{C}$ y 33 a 67 \% HR mínima (Montes-García et al., 2009).

Cuadro 3. Comparación de medias de las interacciones de primer orden entre genotipo, fecha de siembra, fungicida y severidad de $C$. africana, en variables relacionadas con la calidad fisiológica y física de la semilla de sorgo.

\begin{tabular}{|c|c|c|c|c|c|c|c|}
\hline Interacción & $\mathrm{PN}$ & PA & PSM & $\mathrm{V}$ & VIA & P100S & $\mathrm{C}$ \\
\hline & \multicolumn{7}{|c|}{$G \times F$} \\
\hline A1 x F1 & $87 \mathrm{a}$ & $6 \mathrm{a}$ & $7 \mathrm{~b}$ & $78 \mathrm{a}$ & 93 a & $2.133 \mathrm{a}$ & $60.6 \mathrm{~b}$ \\
\hline A9 x F1 & $84 \mathrm{ab}$ & $5 \mathrm{ab}$ & $11 \mathrm{ab}$ & $76 \mathrm{ab}$ & $89 \mathrm{ab}$ & $2.129 \mathrm{a}$ & $73.1 \mathrm{ab}$ \\
\hline $\mathrm{A} 1 \times \mathrm{F} 2$ & $85 \mathrm{ab}$ & $6 \mathrm{ab}$ & $9 a b$ & $76 \mathrm{ab}$ & $90 \mathrm{ab}$ & $2.000 \mathrm{~b}$ & $69.2 \mathrm{ab}$ \\
\hline $\mathrm{A} 9 \times \mathrm{F} 2$ & $81 \mathrm{~b}$ & \multicolumn{5}{|c|}{$\mathrm{G} \times \mathrm{S}$} & $81.7 \mathrm{a}$ \\
\hline A1x S0 & $91 \mathrm{a}$ & $2 \mathrm{c}$ & $7 \mathrm{~b}$ & $80 \mathrm{a}$ & 98 a & $2.122 \mathrm{a}$ & $60.7 c$ \\
\hline A1 x S10 & $87 \mathrm{ab}$ & $4 \mathrm{bc}$ & $9 \mathrm{ab}$ & $79 \mathrm{a}$ & $92 \mathrm{ab}$ & $2.164 \mathrm{a}$ & $58.2 \mathrm{c}$ \\
\hline A1 x S50 & $85 \mathrm{ab}$ & $5 \mathrm{bc}$ & $9 \mathrm{ab}$ & $77 \mathrm{a}$ & $90 \mathrm{ab}$ & $1.910 \mathrm{a}$ & $69.7 \mathrm{bc}$ \\
\hline A9 x S0 & $86 \mathrm{ab}$ & $5 \mathrm{bc}$ & $9 \mathrm{ab}$ & $74 \mathrm{a}$ & $91 \mathrm{ab}$ & $2.026 \mathrm{a}$ & $71.2 \mathrm{bc}$ \\
\hline A9 x S10 & $83 \mathrm{~b}$ & $6 \mathrm{~b}$ & $11 \mathrm{ab}$ & $74 \mathrm{a}$ & $88 \mathrm{~b}$ & $2.121 \mathrm{a}$ & $79.9 \mathrm{ab}$ \\
\hline A9 x S50 & \multicolumn{6}{|c|}{$\mathrm{G} \times \mathrm{Q}$} & $89.3 \mathrm{a}$ \\
\hline A1x CQ & $87 \mathrm{a}$ & $4 \mathrm{bc}$ & $8 \mathrm{~b}$ & $77 \mathrm{ab}$ & 92 a & $2.059 \mathrm{a}$ & $61.1 \mathrm{bc}$ \\
\hline A1 x SQ & $88 \mathrm{a}$ & $3 \mathrm{c}$ & $9 a b$ & $79 \mathrm{a}$ & 93 a & $2.071 \mathrm{a}$ & $59.3 \mathrm{c}$ \\
\hline A9 x CQ & $81 \mathrm{~b}$ & $7 \mathrm{ab}$ & $12 \mathrm{a}$ & $72 \mathrm{c}$ & $86 \mathrm{~b}$ & $2.082 \mathrm{a}$ & $75.3 \mathrm{ab}$ \\
\hline A9 x SQ & \multicolumn{6}{|c|}{$\mathrm{F} \times \mathrm{S}$} & $84.7 \mathrm{a}$ \\
\hline F1 x S0 & $91 \mathrm{a}$ & $3 d$ & $6 \mathrm{~b}$ & $82 \mathrm{a}$ & 96 a & $2.220 \mathrm{a}$ & $57.9 \mathrm{~d}$ \\
\hline $\mathrm{F} 1 \times \mathrm{S} 10$ & $89 a b$ & $4 \mathrm{~cd}$ & $7 \mathrm{~b}$ & $80 a b$ & $94 \mathrm{ab}$ & $2.314 \mathrm{a}$ & $53.6 \mathrm{~d}$ \\
\hline F1 x S50 & $82 \mathrm{~cd}$ & $9 \mathrm{a}$ & $9 \mathrm{ab}$ & $73 \mathrm{bc}$ & $87 \mathrm{~cd}$ & $1.978 \mathrm{a}$ & $66.0 \mathrm{~cd}$ \\
\hline F2 x S0 & $85 \mathrm{bc}$ & $5 \mathrm{bcd}$ & $10 \mathrm{ab}$ & $75 \mathrm{bc}$ & $90 \mathrm{bc}$ & $1.926 \mathrm{~b}$ & $73.9 \mathrm{bc}$ \\
\hline $\mathrm{F} 2 \times \mathrm{S} 10$ & $81 \mathrm{~cd}$ & $6 a b c$ & $13 \mathrm{a}$ & $73 \mathrm{bc}$ & $86 \mathrm{~cd}$ & $1.920 \mathrm{~b}$ & $84.0 \mathrm{ab}$ \\
\hline F2 x S50 & \multicolumn{7}{|c|}{$Q \times S$} \\
\hline CQ x S0 & 89 a & $4 \mathrm{~b}$ & $7 \mathrm{~b}$ & $79 a$ & $94 \mathrm{a}$ & $2.113 \mathrm{a}$ & $62.7 \mathrm{~b}$ \\
\hline CQ x S10 & $85 \mathrm{ab}$ & $5 \mathrm{ab}$ & $10 a b$ & $75 \mathrm{c}$ & $90 \mathrm{ab}$ & $2.155 \mathrm{a}$ & $65.8 \mathrm{~b}$ \\
\hline CQ x S50 & $78 \mathrm{c}$ & $8 \mathrm{a}$ & $14 \mathrm{a}$ & $70 \mathrm{c}$ & $83 \mathrm{c}$ & $1.946 \mathrm{a}$ & $83.6 \mathrm{a}$ \\
\hline SQ x S0 & $86 a b$ & $4 \mathrm{~b}$ & $10 \mathrm{ab}$ & $78 \mathrm{ab}$ & $92 \mathrm{ab}$ & $2.036 \mathrm{a}$ & $69.1 \mathrm{~b}$ \\
\hline $\mathrm{SQ} \times \mathrm{S} 10$ & $85 \mathrm{ab}$ & $5 \mathrm{ab}$ & $10 \mathrm{ab}$ & $78 \mathrm{ab}$ & $90 \mathrm{ab}$ & $2.131 \mathrm{a}$ & $71.9 \mathrm{ab}$ \\
\hline SQ x S50 & 82 bc & $8 \mathrm{a}$ & $10 \mathrm{ab}$ & $73 \mathrm{bc}$ & $88 \mathrm{bc}$ & $1.992 \mathrm{a}$ & $74.9 \mathrm{ab}$ \\
\hline
\end{tabular}

Medias con letras iguales no son estadísticamente diferentes en una columna y por interacción (Tukey, 0.05). G = Línea androestéril A1 y A9; F1 y F2 = Fecha de siembra del 5 de mayo y 10 de junio del 2006; S0, S10 y S50 = Grado de severidad 0, 10 y $50 \%$; CQ y SQ = Con y sin fungicida; PN, PA y PSM = Porcentaje de plántulas normales, anormales y semillas muertas; V = Vigor (\%); VIA = Viabilidad (\%); P100S = Peso de 100 semillas $(\mathrm{g}) ; \mathrm{C}=$ Conductividad eléctrica $\left(\mu \mathrm{s} \mathrm{g}^{-1}\right)$.

Cuadro 4. Correlaciones entre las variables fisiológicas y físicas de la semilla y número de colonias fungosas.

\begin{tabular}{|c|c|c|c|c|c|c|c|c|}
\hline & PN & PA & PSM & VIA & $\mathrm{V}$ & P100S & $\mathrm{C}$ & TCF \\
\hline PN & & 0.127 & $-0.929 *$ & 0.990* & $0.981 *$ & 0.633 & $-0.901 *$ & 0.075 \\
\hline PA & & & -0.188 & -0.102 & -0.233 & -0.180 & -0.100 & 0.441 \\
\hline PSM & & & & $-0.944 *$ & $-0.958 *$ & $-0.783^{*}$ & $0.935^{*}$ & -0.336 \\
\hline VIA & & & & & $0.972 *$ & 0.681 & $-0.928 *$ & 0.161 \\
\hline V & & & & & & 0.684 & $-0.889 *$ & 0.077 \\
\hline P100S & & & & & & & $-0.827 *$ & 0.507 \\
\hline $\mathrm{C}$ & & & & & & & & -0.460 \\
\hline
\end{tabular}

PN, PA y PSM = Porcentaje de plántulas normales, anormales y muertas; VIA = Viabilidad $(\%)$; V = Vigor; P100S = Peso de 100 semillas (g); $\mathrm{C}=$ Conductividad $(\mu \mathrm{s} / \mathrm{g})$ y $\mathrm{TCF}=$ Total de colonias fungosas. $* * \mathrm{P}=<0.01$ 
Según Montes-Belmont et al. (2002), en el Estado de Morelos, México, bajo incidencia natural, las condiciones óptimas inductivas de la enfermedad fueron $25{ }^{\circ} \mathrm{C}$ de temperatura media y máxima de $28{ }^{\circ} \mathrm{C}$ con $96 \%$ HR diurna y $86 \%$ nocturna. En el presente experimento, las condiciones de humedad y temperatura fueron favorables para inducir la enfermedad, pues de floración a cosecha el promedio de temperatura y humedad relativa máximas, mínimas y medias de cada fecha de siembra fueron: 29.5 , 6.8 y $18.2{ }^{\circ} \mathrm{C}$, con $99.3,43.5$ y $71.4 \%$ de HR $(5$ de mayo); $28.7,4.8$ y $16.9{ }^{\circ} \mathrm{C}$, con $98.5,38.6$ y $68.5 \%$ de HR (10 de junio).

\section{Sanidad de la semilla}

En total, en este estudio se detectaron 334 colonias fungosas, distribuidas de la siguiente forma: Alternaria spp. $(262=78 \%)$, Fusarium spp. $(66=19 \%)$ y Aspergillus spp. $(6=3 \%)$ (Cuadro 5). Las especies identificadas fueron: $A$. tenuissima, $A$. tenuis, $F$. oxysporum, $F$. thapsinum, $A$. flavus y $A$. niger. Esta micoflora secundaria no está asociada con la presencia de ergot pues esas especies también se ha encontrado en semilla proveniente de lotes correspondientes a los ciclos 1995 y 1996 de Valles Altos (Cisneros-López et al., 2007); es decir, en años previos al ingreso de $C$. africana a México ocurrida en 1997 (Aguirre et al., 1997). En México, sólo se han investigado algunos hongos postcosecha, como A. flavus Link. Fr., porque están asociados con la producción de micotoxinas que afectan el grano de maíz (Díaz-Franco y Montes-García, 2008). Shekaramúrthy et al. (1994) reportaron en semilla de sorgo que $A$. tenuissima y $A$. tenuis tuvieron mayor incidencia respecto a otros hongos de campo ( $F$. moniliforme) y almacén (Aspergillus spp.).
La aplicación de Thiram ${ }^{\circledR}$ redujo en $31 \%$ el total de colonias fungosas y en $42 \%$ las de Alternaria ( $\mathrm{P}<$ 0.05), respecto a la semilla sin fungicida. No se encontraron diferencias significativas con respecto a Fusarium y Aspergillus, posiblemente porque su incidencia fue menor (Cuadro 5). Se ha reportado que Thiram ${ }^{\circledR}$ es un fungicida efectivo en el control de hongos en la semilla de sorgo, como A. alternata, A. tenuisssima y $F$. moniliforme (Vidhyasekaran, 1983; Shekaramúrthy et al., 1994). La diferencia del efecto del fungicida entre especies de hongos puede atribuirse al sitio donde infectan la semilla; Alternaria spp. es infectada en su exterior mientras que Fusarium spp. también puede ser infectado en su interior (Singh, 1983), en función del momento en que ocurra la infección post-antesis (Little, 2000). Estos resultados pueden atribuirse al modo de acción del Thiram ${ }^{\circledR}$, pues es un fungicida de contacto que actúa en la superficie de la semilla donde inhibe la germinación de esporas y el crecimiento del micelio de hongos (Lucas y Dickinson, 1998).

Las semillas provenientes de panojas con 10 y $50 \%$ de severidad de $C$. africana presentaron de 1.8 y 2.6 veces $(\mathrm{P}<0.05)$ más colonias fungosas que las semillas del testigo sin daño aparente (Cuadro 5), pues los hongos saprófitos tuvieron más sustrato para colonizar porque los exudados de Claviceps africana están constituidos por oligosacáridos (Mantle y Bogo, 2002). Estos resultados corroboran que el ergot, como patógeno primario, favorece la presencia de micoflora secundaria que con frecuencia se presenta en la semilla de sorgo en postcosecha (Little, 2000).

Cuadro 5. Número de colonias fungosas en la semilla de sorgo en cada tratamiento.

\begin{tabular}{|c|c|c|c|c|c|c|c|c|c|c|c|}
\hline \multirow[b]{2}{*}{ Severidad \% } & \multicolumn{3}{|c|}{ Con fungicida (CQ) } & \multicolumn{4}{|c|}{ Sin fungicida (SQ) } & \multirow[b]{4}{*}{$\begin{array}{c}\text { Suma } \\
\text { SO }\end{array}$} & & & \multirow{4}{*}{ TCF } \\
\hline & Alternaria & Fusarium & Aspergillus & & \multirow[t]{2}{*}{ Alternaria } & Fusarium & Aspergillus & & \multirow{2}{*}{\multicolumn{2}{|c|}{ Subtotal }} & \\
\hline & & & & 5 de mayo & & & & & & & \\
\hline & $\mathrm{A} 1$ & A1 A9 & A1 A9 & $\begin{array}{c}\text { Suma } \\
\text { CQ }\end{array}$ & A1 A9 & A1 A9 & A1 A9 & & A1 & A9 & \\
\hline 0 & 5 & 3 & 0 & 12 & 3 & 2 & 2 & 15 & 15 & 10 & $25^{*}$ \\
\hline 10 & 18 & 8 & 0 & 34 & 212 & 3 & 0 & 33 & 54 & 15 & $67^{*}$ \\
\hline 50 & 14 & 3 & 0 & 25 & $25 \quad 13$ & 0 & 0 & 39 & 43 & 21 & $64^{*}$ \\
\hline Subtotal & $37 \quad 10$ & 148 & 02 & 5120 & $4920 *$ & $10 * 5$ & 2 & $6126^{*}$ & 112 & 46 & \\
\hline TCF & 47 & 22 & 2 & $\begin{array}{c}\mathbf{7 1}^{*} \\
10 \text { de junio }\end{array}$ & 69 & 15 & 3 & $87 *$ & & & 158 \\
\hline 0 & 6 & 2 & 0 & 17 & $\begin{array}{ll}13 & 8\end{array}$ & 2 & 0 & 23 & 23 & 17 & $40 *$ \\
\hline 10 & 4 & 2 & 0 & 14 & $17 \quad 18$ & 3 & 0 & 41 & 26 & 29 & $55^{*}$ \\
\hline 50 & $\begin{array}{ll}5 & 24\end{array}$ & 4 & 0 & 34 & $14 \quad 27$ & 33 & 0 & 47 & 26 & 55 & $81 *$ \\
\hline Subtotal & $14 \quad 35$ & 87 & 10 & 2342 & $4453^{*}$ & $8 * 6$ & $0 \quad 0$ & $52 \quad 9^{*}$ & 75 & 101 & \\
\hline TCF & 49 & 15 & 1 & $65^{*}$ & 97 & 14 & 0 & $111^{*}$ & 189 & 147 & 176 \\
\hline GTCF & 96 & 37 & 3 & & 166 & 29 & 3 & 138 & 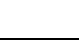 & & 334 \\
\hline
\end{tabular}


El número total de colonias obtenidas en la fecha de siembra del 5 de mayo (Lote 1$)$ no fue diferente $(\mathrm{P}<$ 0.05) del de la siembra del 10 de junio (Lote 2) (Cuadro 5 ), a pesar de que en la primera fecha hubo mayor humedad durante postfloración ( 22 d con lluvia y 85.9 $\mathrm{mm}$ de precipitación, con $71.4 \%$ de humedad relativa promedio) que en la fecha del 10 de junio ( $11 \mathrm{~d}$ de lluvia con $56.3 \mathrm{~mm}$ y $68.5 \% \mathrm{HR}$ ). Como las panojas de las líneas androestériles se embolsaron para evitar daños por pájaros y se mantuvieron en esta condición $41 \mathrm{~d}$ en el Lote 1 y $45 \mathrm{~d}$ en el Lote 2 , es posible que ésta sea la causa de que incluso las panojas sin daño aparente de $C$. africana hayan presentado patógenos secundarios.

Las correlaciones entre el número de colonias formadas por Alternaria, Aspergillus y Fusarium y el porcentaje de plántulas normales, anormales, semillas muertas, viabilidad, vigor, P100S y conductividad eléctrica, no fueron significativas, lo que se puede atribuir a que la mayor parte de micoflora secundaría colonizó la superficie de la semilla y no penetró en interior, ni dañó al embrión. En la semilla de sorgo el carácter patogénico de estos hongos está más asociado con la colonización del embrión que la del pericarpio (McLaren et al, 2002), pero en este experimento no se hizo esta evaluación.

\section{CONCLUSIONES}

El genotipo y la fecha de siembra tuvieron mayor efecto $(\mathrm{P} \leq 0.05)$ que el fungicida y la severidad del daño de $C$. africana (S) en la calidad física y fisiológica de la semilla. $\mathrm{Su}$ efecto superó al de las interacciones en las siete variables. La línea androestéril A1 tuvo mejor calidad, germinación y vigor que la línea A9, con menos semillas muertas y conductividad eléctrica. El lote sembrado el 5 de mayo del 2006 presentó mayor germinación de plántulas normales, viabilidad, vigor, tamaño de semilla y menor conductividad eléctrica, que el lote sembrado el 10 de junio. En general, la calidad sanitaria de la semilla fue afectada por el grado de severidad de la panoja en campo, pero no por la aplicación de fungicida a la semilla.

\section{BIBLIOGRAFÍA}

Aguirre R J I, H Williams, G N Montes, M Cortinas (1997) First report of sorghum ergot caused by Sphacelia sorghi in México. Plant Dis. 81:31.

Bandyopadhyay R, L K Mughogho, S K Manohar, M V Satyanarayana (1990) Stroma development, honeydew formation and conidial production in Claviceps sorghi. Phytopatology 80:812-818.

Bandyopadhyay R, D E Frederickson, N W McLaren, G N Odvody, M J Ryley (1998) Ergot: A new disease threat to sorghum, in the Americas and Australia. Plant Dis. 82:356367.
Bekendam, J, R Grob (1980) Manual para Evaluación de Plántulas. In: Análisis de Germinación. Instituto Nacional de Semillas y Plantas de Vivero. Estación de Ensayo de Semillas Madrid España.130 p.

Castor L L, R A Frederiksen (1981) Fusarium head blight occurrence and effects on sorghum yield and grain characteristic in Texas. Plant Dis. 64:1017-1019.

Cisneros-López M E, L E Mendoza-Onofre, G Mora-Aguilera, L Córdova-Téllez, M Livera-Muñoz (2007) Cold tolerant sorghum hybrids and parental lines. I: Seed quality and its effects on seedling establishment. Agrociencia 41:45-55.

Cruz-Pérez A B, V A González-Hernández, $M$ del C MendozaCastillo, M L Ortega-Delgado (2002) Marcadores fisiológicos de la tolerancia al envejecimiento de semillas de maíz. Agrociencia 37:371-381.

Dabkeviciius Z, R Mikaliūnaitê (2006) The effect of fungicidal seed treaters on germination of rye ergot (Claviceps purpurea (Fr.) Tul.) sclerotia and on ascocarp formation. Crop Prot. 25:677683.

Dahlberg J A, G L Peterson, G N Odvody, M Bonde (1999) Inhibition of germination and sporulation of Claviceps africana from honeydew encrusted sorghum with seed treatment fungicides. Crop Prot. 18:235-238.

Díaz-Franco A, N Montes-García (2008) La fitopatología en la región semiárida de Tamaulipas México: Reseña histórica. Rev. Mex. Fitopatol. 26:62-70.

Frederiksen R A, G N Odvody (2000) Compendium of Sorghum Diseases. APS. Press. St. Paul, Minnesota, USA, 78 p.

Frederiksen R A, G N Odvody (2003) Inhibition of germination of sphacelial of Claviceps africana following treatment of seedsphacelial admixtures with captan. Crop Prot. 22:95-98.

Futrell M C, O J Webster (1966) Host range and epidemiology of the sorghum ergot organism. Plant Dis. Rep. 50:828-831.

Hernández-Martínez, M, L E Mendoza-Onofre, P RamírezVallejo, E Cárdenas-Soriano (2006) Incidence and severity of ergot (Claviceps africana Frederickson, Mantle and de Milliano) in two male-sterile sorghum lines as a function of distance from pollen source and its impact on seed production and quality. Seed Sci. Technol. 34:393-402.

ISTA, International Seed Testing Association (1999) International Rules for Seed Testing. International Seed Testing Association (Ed). Zurich, Switzerland. 321 p.

Little C R (2000) Plant responses to early infection events in sorghum grain mold interactions. In: Technical and Institutional Options. Sorghum Grain Mold Management Proceedings of an International Consultation. 18-19 May 2000, ICRISAT, Patancheru, India. A Chandrashekar, R Bandyopadhayay, A J Hall (eds). Patancheru 502324, Andhra Pradesh, India. pp:169-182.

Lucas A J, C H Dickinson (1998) Plant Pathology and Plant Pathogens. 3rd ed. Blackwell Pub. Boston, Massachusetts, USA. $274 \mathrm{p}$.

Mantle P G, A Bogo (2002) Biosyntesis of bioactive honeydew oligosaccharides by sorghum ergot pathogens. In: Sorghum and Millets Diseases. J F Leslie (ed). Iowa State Press, Ames, IA. USA. pp: 91-94.

Marsans G J (1987) Manejo y Conservación de Granos. Ed. Hemisferio Sur. Argentina. $266 \mathrm{p}$

McLaren N W (1993) Effect of sugary disease exudates on germination, seedling development and predisposition to seedling diseases of sorghum (Sorghum bicolor). South Africa Tydskrif Plant Grond 10:12-16.

McLaren N W, J Saayman, J Benade and M van der Walt (2002) Evaluation of reduced sorghum seed germination. In: Sorghum and Millet Diseases. J F Leslie (ed). Iowa State Press, Ames, IA. USA. pp:267-268. 
McLaren N W, F C Wehner (1990) Relationship between climatic variables during early flowering of sorghum and incidence of sugary disease caused by Sphacelia sorghi. J. Phytopathol. 130:82-88

Meinke, H, M Ryley (1997) Effects of sorghum ergot on grain sorghum production: a preliminary climatic analysis. Aust. J. Agric. Res. 48:1241-1247.

Montes-Belmont R, I Mendez-Ramírez, E Flores-Moctezuma (2002) Relationship between sorghum ergot, sowing dates and climatic variables in Morelos, México. Crop Prot. 21:899905.

Montes-García N, L K Prom, H Williams-Alanis, T Isakeit (2009) Effect of temperature and relative humidity on sorghum ergot development in northern México. Australasian Plant Pathol. 38:632-637.

Navarrete-Maya R， J Acosta-Gallegos (1999) Reacción de variedades de fríjol a Fusarium spp. y Rhizoctonia solanni en el Altiplano de México. Agron. Mesoam. 10:37-46.

Narasimhulu T, P K Rao (1989) Effect of seed treatment with insecticides and fungicides on the germination of peanut seed. Seed Res. 17:159-163.

Odvody G N, D E Frederickson, T Isakeit, N Montes-García, J A Dahlberg, G L Peterson (2002) Quarantine issues arising from contamination of seed with ergot: An update. In Sorghum and Millets Diseases. J F Leslie (ed). Iowa State Press, Ames, IA. USA. pp:123-127.

Peña R A, J D Eastin, S D Kachman, F Zavala G (2001) Herencia de la duración del periodo y tasa de llenado de grano en sorgo. Rev. Fitotec. Mex. 24:171-178.

Pérez M M, A Hernández L, F V González C, G García de los Santos, A Carballo C, T Roque R, M Tovar G (2006) Seed size and its relationship with physiological quality in forage maize cultivars. Agric. Téc. Méx. 32:341-352

Powell A (1988) Seed vigor and field establishment. Adv. Res.Techno. Seeds 11:29-61.

SIAP, Sistema de Información Agropecuaria (2008) Panorama internacional para el sorgo. Secretaría de Agricultura, Ganadería, Desarrollo Rural, Pesca y Alimentación. Boletín Mensual al 30 de diciembre del 2007. 20 p.
SAS Institute (2002) SAS/SAT. Versión 9.1. SAS Inst. Cary. NC. USA. Disponible en: http://www.colpos.mx/servicios/sas/ indexs.htm. (Octubre 2008)

Singh D (1983) Histopathology of some seed-borne infections: A review of recent investigations. Seed Sci. Technol. 11:651663.

Singh A E, V G Makne (1985) Correlations studies on seed viability and seedling vigor relation to seed size in sorghum (Sorghum bicolor). Seed Sci. \& Technol. 13:139-142.

Shekaramúrthy S, K L Paktar, S A Shetty, H S Prakash, H. S Shetty (1994) Effect of thiram treatment on sorghum seed quality in relation to accelerated ageing. Seed Sci. Technol. 22:607-617.

SNICS, Servicio Nacional de Inspección y Certificación de Semilla (1987) Normas para la Certificación de Semillas. Servicio Nacional de Inspección y Certificación de Semillas. México, D.F. 80 p.

Valadez-Gutiérrez J, L E Mendoza-Onofre, H Vaquera-Huerta, L Córdova-Téllez, Ma del C Mendoza-Castillo, G García-de los Santos (2006) Flowers thinning, seed yield and post anthesis dry matter distribution in sorghum. Agrociencia 40:303-314.

Veselova T V, V A Veselovsky (2003) Investigation of atypical germination changes during accelerated ageing of pea seed. Seed Sci. Technol. 31:517-530.

Vidhyasekaran P (1983) Control of Fusarium moniliforme infection in sorghum seed. Seed Sci. \& Technol. 11:435-439.

Wang Y R, L Yu, Z B Nan, Y L Liu (2004) Vigor test used to rank seed lot quality and predict field emergence in four forage species. Crop Sci. 44:535-541.

Warham E J, L D Butler, B C Sutton (1994) Ensayos para la Semilla de Maíz y de Trigo. Manual de Laboratorio. CIMMYT. México, D. F. 84 p.

Wen-Shi W, C Kuang-Che (1990) Relationships between seed health, seed vigour and the performance of sorghum in the field. Seed Sci. Technol. 18:713-719.

Woltz J, D M Tekrony, D B Egli (2006) Corn seed germination and vigor following freezing during seed development. Crop Sci. 46:1526-1535 